\title{
Encyclopedia of cells in early pregnancy
}

A well-formed placenta is crucial for a healthy pregnancy, and early placental dysfunction can lead to adverse outcomes in late pregnancy, such as stillbirth and preeclampsia. Placentation involves both fetal and maternal cells, but the specific cell types involved and how they interact to form the placenta, particularly in the first trimester, are not well understood. Now, two papers report singlecell RNA-seq analyses from first-trimester human placentas that identify new cellular subtypes and shed light on maternal-fetal cell communication in early pregnancy ${ }^{1,2}$.

Soon after conception, the lining of the uterus differentiates to form the decidua, the maternal part of the placenta that interfaces with the fetus. Decidualization enables invasion by fetal extravillous trophoblast (EVT) cells, which transform maternal spiral arteries to function effectively to nourish the growing fetus. Fetal EVTs also interact with maternal decidua and immune cells to enable the uterus to accept both the placenta and the fetus. In mammals, reproduction and immunology are evolutionarily divergent, and placentation is highly variable. Therefore, placentation must be studied in humans to tackle pregnancy problems, and single-cell RNA-seq has enabled a more precise lens.

The first step in developing a comprehensive understanding of human placentation is to precisely define fetal and maternal placental cell types. Single-cell transcriptomes have been generated for placental and decidual tissues at the end of gestation ${ }^{3,4}$, and, more recently, RNA-seq analysis of 1,567 fetal placental cells from first- and second-trimester placentas identified 14 placental cell subtypes ${ }^{5}$. By focusing in on samples from 6 to 14 weeks' gestation and analyzing higher numbers of both fetal and maternal single cells, Vento-Tormo et al. ${ }^{1}$ and Suryawanshi et al. ${ }^{2}$ are able to present a more comprehensive survey of cell types and to start to identify mechanisms that underlie healthy placentation.

Both groups relied on single-cell transcriptomics to map out early human placentation. Suryawanshi et al. ${ }^{2}$ used Drop-seq and the commercial droplet Chromium platform to examine transcriptomes of more than 20,000 fetal and maternal placental cells. VentoTormo et al. ${ }^{1}$ used droplet Chromium and plate-based (Smart-seq2) methods and analyzed more than 70,000 fetal and maternal

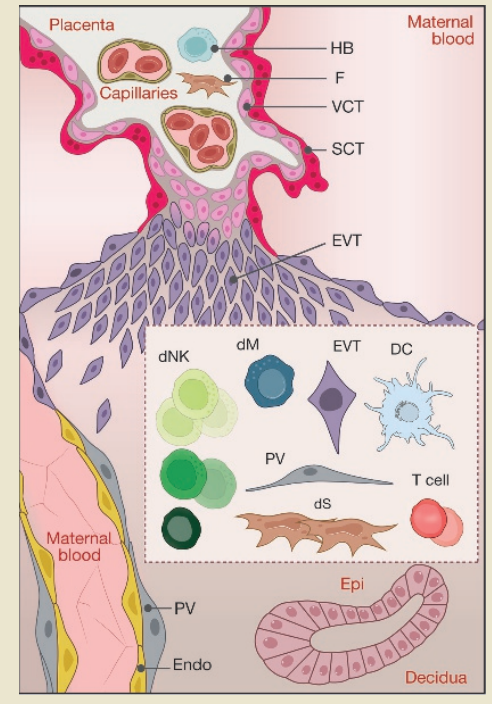

Reprinted with permission from ref. 1.

placental cells, as well as maternal blood cells. Both studies clustered cells to identify subtypes and identified cells as maternal or fetal using genetic markers. According to Hongmei Wang, director of the State Key Laboratory of Stem Cell and Reproductive Biology at the Institute of Zoology, Chinese Academy of Science, Beijing, who was uninvolved with either paper, "the strength of these studies is in studying a huge amount of cells at the fetal-maternal interface, which is very important for the establishment and maintenance of pregnancy."

In both reports, the authors set about interpreting their atlases of early placental cell types using computational analyses to identify interactions that might be biologically relevant to placentation. Understanding which cell-cell interactions occur, and when, could reveal mechanisms of placentation.

Suryawanshi et al. ${ }^{2}$ manually curated ligand-receptor pairs and integrated this information with cell type-specific expression levels to output cell-to-cell interaction diagrams. Their in silico approach enabled the identification of potentially biologically relevant interactions.

To derive biological insights from their single-cell datasets, Vento-Tormo et al. ${ }^{1}$ developed a computational framework named CellPhone to predict cell-typespecific ligand-receptor complexes. The outputs of the CellPhone algorithm are collated in an open database, CellPhoneDB, that integrates information from UniProt, Ensembl, PDB, the IMEx consortium and IUPHAR to derive as many leads as possible in terms of understanding which cell types interact and how. The authors used CellPhoneDB to assess potential interactions among cell types identified in their study. Importantly, they validated their in silico predictions with spatial in situ analyses (immunohistochemistry as well as multiplexed single-molecule fluorescence in situ hybridization and marker-assisted flow cytometry) to map mechanisms that underlie human placentation.

The resolution afforded by the combination of single-cell transcriptomics, in silico interaction analyses and orthogonal experimental validation enabled Vento-Tormo et al. ${ }^{1}$ to delineate distinct subtypes of perivascular, stromal and natural killer (NK) cells. Notably, they define three new subsets of decidual NK cells and predict that these cells constrain the extent of trophoblast invasion and mediate immunomodulatory pathways in early pregnancy. As Wang notes, "many different type of cells are involved in maternal-fetal communication, including trophoblast cells, decidual stromal cells and immune cells, and the types and subtypes of cells may change during the course of pregnancy. The study by Vento-Tormo et al. ${ }^{1}$ not only maps the cells that are there, it generates hypotheses about placental development and in particular the roles of NK cell subtypes."

Although the use of single-cell RNA-seq to study human placentation is just beginning, the encyclopedia of early placental cell types and their interactions has already produced substantial new insights into how the function of the maternal immune system is affected by cells from the developing placenta. Establishing single-cell atlases for placentation is an enormous step forward for understanding not only pregnancy but women's health in general.

\section{Susan Jones, Senior Editor}

1. Vento-Tormo, R. et al. Nature 563, 347-353 (2018).

2. Suryawanshi, H. et al. Sci. Adv. 4, eaau4788 (2018)

3. Pavličev, M. et al. Genome Res. 27, 349-361 (2017).

4. Tsang, J.C.H. et al. Proc. Natl. Acad. Sci. USA 114 E7786-E7795 (2017)

5. Liu, Y. et al. Cell Res. 28, 819-832 (2018). 